\title{
Next Generation Avionics System Of Future Launch Vehicle
}

\author{
Guangping Qi ${ }^{*}$, Ran Duan, Haifeng Sun, Hu Zhou, Zongyou Xiang, Wenting li \\ Beijing Aerospace Automatic Control Institute,Beijing, China \\ P00000000@sina.com
}

\begin{abstract}
Keywords: Avionics system; launch vehicle; next generation
Abstract: One new conception avionics system of launch vehicle is proposed according to the future technology trend. Aimed at efficient, low-cost and reliable space transportation system, the entire avionics system scheme is proposed innovatively in this paper. There are some characteristics in the avionics system as following: 1) The rocket-borne devices are miniaturization and standardization designed. 2) The vehicle performs checkout intelligently and autonomously. 3) The connection between rocket and ground facilities is simplified remarkably. 4) The launch facilities and operation is compacted greatly. 5) Launch control and management is mobile for the wireless networks utilized. 6) The cloud technology is used to build backbone network in the launch system. The onboard devices are designed to be highly intelligent so that the vehicle performs checkout autonomously. The rocket is connected to the ground support facilities, via a high-speed network, with rare cables. On the other hand, because many checkout equipments are fixed to rocket body, lots of ground support equipments can all be eliminated and the associated set-up time and the number of personnel involved can be reduced. Moreover, all the equipments are connected via a secured high-speed network, which make it possible to conduct the launch control anytime, anywhere in the world simply by using a single laptop computer or portable device such as mobile phone, pad etc. This means having an ultimate launch control system which is independent of launch sites. In the next generation launch system, a simpler launch system allows the frequent launch by largely-reducing operational costs through enhancing aspects of operational efficiency launch systems are improved and simplified. These innovative concepts will be a role model for future launch vehicles.
\end{abstract}

\section{Introduction}

The first flight of Japan's Epsilon launch vehicle, the newest version solid propulsion rocket, makes a great response in space domain on 14, Sep, 2013. Many original design concepts, such as the highly intelligent autonomous checkout system and the mobile launch control, are innovatively realized [1-2].

The lift-off of Epsilon can be executed in less than 6 days after the rocket erected on launch pad. Moreover, JAXA has been conducting intensive researches on a next generation Epsilon to launch a more powerful and lower cost version Epsilon (E1) in 2017.

America, Russia and Europe also have new programs to research and develop the next generation commercial launch vehicle aimed at low cost.

The new launch vehicle aims at low cost and fast launch, which is mainly decided by the avionics system.

With the development of electronic and communication technology, one scheme for next generation avionics system of future launch vehicle is proposed in this paper.

\section{The Top Design For Next Generation Avionics System}

The Design Conception. In order to cut down the cost and improve the launch frequency, some new design ideas are needed to change the traditional work flow. Firstly, compared with traditional launch vehicle, more intelligent technologies [3], less operations, less supporting workers are needed in the new transportation system. When the intelligent technology is applied to launch vehicle, the operability and reliability will grow up. It is to say the efficiency of control and operation will be improved. This aspect focuses on highly intelligent autonomous checkout system.

Secondly, the mobile maintenance and launch control based on wireless mobile communication technology will be a trend used in the launch vehicle. The state of rocket and launch can be carried out anywhere if an access port is provided. 
Thirdly, the connection cables between the rocket and ground establishment are simplified if the onboard data transported by wireless communication module and the Lithium battery used as power. There are only very few control signals needed.

Fourth, miniaturization, integration, standardization are characteristic to the onboard units.

Finally, the launch system including equipments and operations will be condensed obviously, for the checkout onboard function is becoming stronger and stronger.

If all the above conceptions become true, the next generation avionics system of the future launch vehicle will make a big advancement.

System Scheme. The avionics system of launch vehicle is made up of the onboard and ground parts. As Fig.1 shows, the onboard avionics system includes power system, control system, telemetry \& safety system. The launch system includes near terminal equipments, such as power cabinet, data transportation box, emergency control and test cabinet etc.

In order to simplify the launch system, one-form mode design conception is used, that is to say, all ground units follow one criterion, make it is possible, traditional function equipments can be integrated into one unit.

Moreover, the cloud computing technology can be introduced in the future launch system. The computer capability will be improved greatly, and each equipment can join into the cloud networks as terminal.

Power system:The power system is mainly made up of Lithium battery, intelligent distributor and power manage and recharging system. All the electric system onboard is powered by the power system, which simplifies the power system greatly.

Because the repeating using character, the battery can be recharged about 2000 times by the online cables. The power managing and recharging system is using to measure the state of battery and recharge the battery when power is low.

The intelligent distributor switches the electronic circuit and sends electric power to special units according to the work flow. And the state of distributor can be gotten from the intelligent interface, which is useful for the malfunction diagnose.

Control system: The control system including Guidance, Navigation and Control (GNC) function, is a kernel and complex system. The fiber channel (FC) is used to exchange the information in control system and between the other systems. The strap-down inertial measuring unit (IMU) is used to build up the inertia coordinate, which is the base of guidance and navigation law. The onboard combination controller $(\mathrm{OBCC})$ gets the inertia information and carries out the control law to control the flying trajectory and attitude of rocket. OBCC including the scheduling control, computing, switch control, BIT etc. functions, is the kernel device of avionics system. There is only one OBCC in every stage.

Telemetry system:Traditional telemetry system measures the parameters of control system is mainly by analog signals, so the coupling between control system and telemetry is heavy. In the future, all the parameters of control system will be given to telemetry by digital bus. There are only few environment parameters such as vibration, pressure, temperature, humidity etc. are measured by telemetry.

The other function of telemetry system will be still reserved.

Launch system:Compared with traditional launch system, the test function is weak for the automatic checkout of onboard control system. The new launch system aimed at launch control and power supply.

The ground equipments of control system and telemetry system are uniformed as standard terminals joined into cloud networks. 


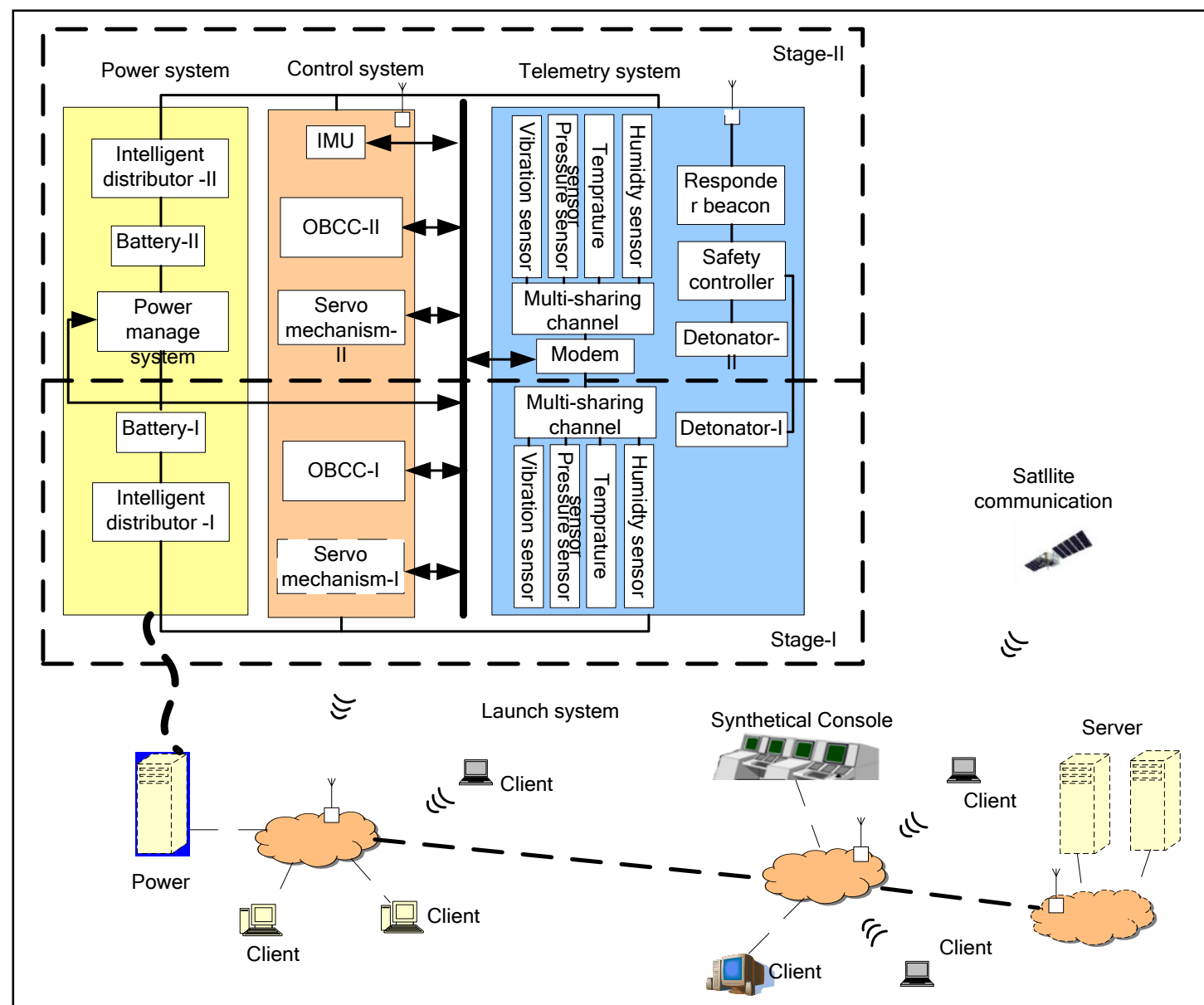

Fig. 1. The functional module of new launch vehicle.

\section{Test Flow Design}

In order to cover all the typical function, all the devices, including hardware and software, must complete a series of experiments to check the function and parameters as Fig.2 shows.The control system and telemetry system must be test completly to find potential quality defects.

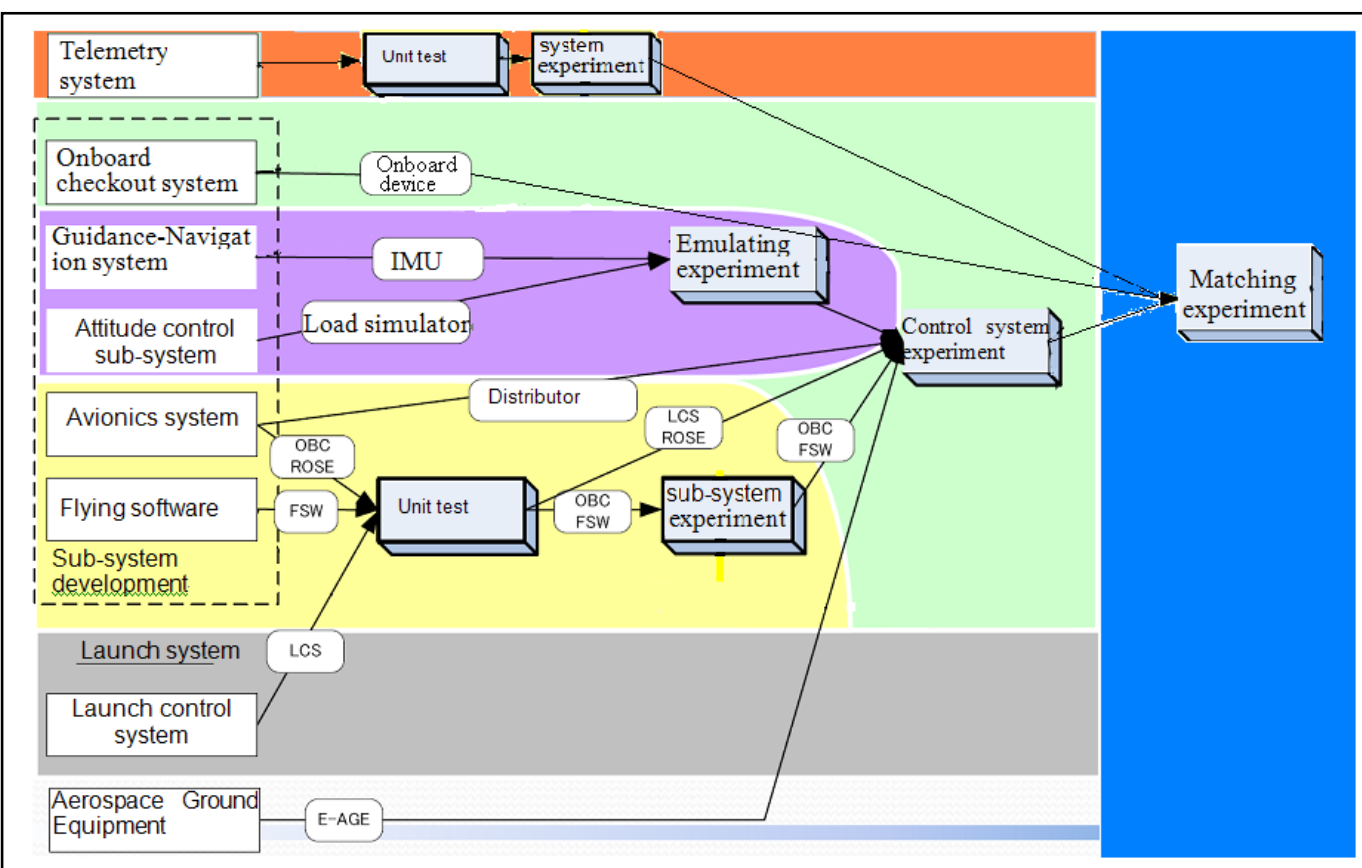

Fig. 2. The test flow of avionics for new launch vehicle. 
Simulation Test. Simulation test is an important step to check up the GNC subsystem.

Usually, the mathematic simulation, half-production simulation and production simulation will be used to simulate the guidance and attitude control function.

Unit Test. All the unit devices have to perform the unit test before joining in the system, which is the reliable working base.

Especially, the unit test of OBCC, servo and IMU are deeply and fully according to the test standards.

Furthermore, condition experiments such as vibration, temperature experiments are used to find any the latency fault. By this way, the quality of production can be guaranteed.

Synthesis Test. Synthesis test mainly includes the sub-system experiment and system experiment. The control system and telemetry system need to complete itself system normally before they connecting each other.

Matching Test. After the synthesis test, every system works well, so they can cooperate with each other.

There maybe have some disturbance when they worked together although each system works well. For example, the telemetry system radiates electromagnetic wave when communication is processing. This electromagnetic wave will affect the electromagnetic condition of control system, which may disturb the work of control system.

Usually, each system has measures to restrain or eliminate the disturbance from other system. In order to check the Electro Magnetic Compatibility (EMC) performance, the matching experiment is necessary.

Onboard Test. All above test can not replace the onboard experiment, because the flying condition is onboard. This test will be carried out after all the device fixed on the rocket body, which leads the electromagnetic condition maybe has changes.

\section{Technology}

Some new technologies are used in the avionics system to improve the whole performance.

Fiber Communication. For the intelligent device becoming more and more, the communication data will increase notably. The data rate of ST-MIL-1553B is $1 \mathrm{Mbps}$, which is weak to transport big data. Compared with ST-MIL-1553B, the FC has high data transportation rate, no electromagnetic leakage, light weight and long communication distance.

So the Fiber Communication (FC) technology with a high data rate 1 4Gbps is a good choice for the data transportation in the next generation avionics.

Wireless Communication. The traditional data transportation for launch vehicle is by wire cable. There are many jobs need to finish before the launch system foundation can be used.

Moreover, the cable limit is not convenient for the install of launch device. The wireless communication is used between the rocket and launch system so that the connection is simplified greatly.

Mobile Launch Control. With the development of wireless communication, the mobile launch control by laptop computer or portable device such as mobile phone, pad etc. is possible.

The mobile communication technology will increase the lay flexibility of launch device even the remote control is possible.

Cloud network. Cloud technology is a new technology, on which can ignore the difference of hardware based. The computing capability will be improved greatly, and each equipment can join into the cloud networks as terminal.

The cloud computer and cloud service are the kernel advantage of cloud network [5]. The cloud computing provides service is based on distributing computers depending on remote server.

Cloud technology is helpful for the malfunction diagnoses.

Software Structure. For the different application, the traditional hardware is manifold, and the software is specially developed according to the hardware config.

In the future, the uniform design is a trend. Operation system (OS) shall be introduced into the avionics to avoid the additional software development. Only the application is changed according to 
different application, and driving program and OS is reused when the software is based on OS. OS improves the software flexible and uniforms the software structure.

\section{Conclusion}

The onboard devices are designed to be highly intelligent so that the vehicle performs checkout autonomously. The rocket is connected to the ground support facilities, via a high-speed network, with rare cables. Lots of ground support equipments can all be eliminated and the associated set-up time and the number of personnel involved can be reduced. Moreover, all the equipments are connected via a secured high-speed wireless network, which make it possible to conduct the launch control anytime, anywhere in the world simply by using a single laptop computer or portable device. This means having an ultimate launch control system which is independent of launch sites. In the next generation launch system, a simpler launch system allows the frequent launch by largely-reducing operational costs through enhancing aspects of operational efficiency launch systems are improved and simplified.

\section{References}

[1] Y. MORITA, T. IMOTO, S. TOKUDOME and H. OHTSUKA, “A Year To Launch: Japan'S Epsilon Launcher And Its Evolution” , 63rd International Astronautical Congress (IAC), Naples, Italy, 2012, Paper No. IAC-12-D2.1.8.

[2] Y. MORITA, T. IMOTO, S. TOKUDOME and H. OHTSUKA, “The first flight of Japan's epsilon launch vechicle” , 64th International Astronautical Congress (IAC), Beijing, China, 2013, Paper No. IAC-13-D2.1.2.

[3] Nikolay Sokolov, Victor Ivanov and Denis Zelenov “The Prospects Of Development And Use Of Intelligent Control Systems For Space Vehicles” , 64th International Astronautical Congress 2013 (IAC), Beijing, China, 2013, Paper No. IAC-13-B26.2.3.x17468.

[4] Ziyu Wang, Haitao Wang and Yongquan Zhu, “Research On Fast Testing Methodsof Launch Vehicle” , 64th International Astronautical Congress (IAC), Beijing, China, 2013, Paper No. IAC-13-D2.p.6.

[5] Justin Cappos, Ivan Beschastnikh, Arvind Krishnamurthy, Tom Anderson. A platform for educational cloud computing, Seattle, WA 98105, U.S.A. 